\title{
Integrated microarray analysis to identify potential biomarkers and therapeutic targets in dilated cardiomyopathy
}

\author{
HAO ZHANG* , JUNYU HUO*, WANYING JIANG and QIJUN SHAN \\ Department of Cardiology, The First Affiliated Hospital of Nanjing Medical University, Nanjing, Jiangsu 210029, P.R. China
}

Received September 23, 2019; Accepted April 9, 2020

DOI: $10.3892 / \mathrm{mmr} .2020 .11145$

\begin{abstract}
Dilated cardiomyopathy (DCM) is a primary cardiomyopathy with high mortality. The aim of the present study was to identify the related genes in DCM. The four expression profiles (GSE17800, GSE21610, GSE42955 and GSE79962) downloaded from the Gene Expression Omnibus (GEO) database were analyzed using RankProd and metaMA R packages to identify differentially expressed genes (DEGs). DEGs were uploaded to the Database for Annotation, Visualization and Integrated Discovery (DAVID), for Gene Ontology (GO) and Kyoto Encyclopedia of Genes and Genomes (KEGG) pathway enrichment analysis. A protein-protein interaction (PPI) network of the DEGs was constructed using the STRING database. In addition, hub genes were identified using the Cytoscape plugin cytoHubba. A mouse DCM model, which established via intraperitoneal injection with doxorubicin (DOX), was used to validate the hub genes. A total of 898 DEGs were identified across the four microarrays. Furthermore, GO analysis demonstrated that these DEGs were mainly enriched in cell adhesion, negative regulation of cell proliferation, negative regulation of apoptotic process and potassium ion transport. In addition, KEGG analysis revealed that DEGs were mainly enriched in the ECM-receptor interaction, the p53 signaling pathway, cardiac muscle contraction and the hypoxia-inducible factor signaling pathway. Proenkephalin (PENK), chordin like 1 (CHRDL1), calumenin (CALU), apolipoprotein L1, insulin-like growth factor binding protein 3 (IGFBP3) and ceruloplasmin (CP) were identified as hub genes in the PPI network. Furthermore, the expression levels of PENK, CHRDL1, IGFBP3, CP and CALU in hearts with DCM were validated using a mouse model. In conclusion, the present study identified six hub genes related to DCM. Therefore, the present results may provide a potential
\end{abstract}

Correspondence to: Dr Qijun Shan, Department of Cardiology, The First Affiliated Hospital of Nanjing Medical University, 300 Guangzhou Road, Nanjing, Jiangsu 210029, P.R. China

E-mail: qjshan@njmu.edu.cn

*Contributed equally

Key words: dilated cardiomyopathy, hub gene, gene expression omnibus, bioinformatics mechanism for DCM involving these hub genes, which may serve as biomarkers for screening and diagnosis in the clinic.

\section{Introduction}

Dilated cardiomyopathy (DCM), a primary cardiomyopathy with significant enlargement of the ventricle (1), is one of the most intractable diseases in the cardiovascular field (2). DCM can progressively develop into severe congestive heart failure and seriously threatens the survival rate of the patient (3). However, due to its unknown etiology and underlying mechanisms, DCM cannot be efficiently treated by existing therapeutic strategies, except for heart transplantation (4). Thus, identifying the specific molecular mechanism of DCM is important to facilitate its diagnosis and treatment, and could improve the prognosis of patients with DCM.

With the development of databases such as The Cancer Genome Atlas and the Gene Expression Omnibus (GEO), genetic research on DCM has become feasible and is currently ongoing (5). Huang et al (6) analyzed 102 samples from the GEO database (GSE5406), and identified module and hub genes of differentially expressed genes (DEGs) that are related to the progression of DCM. In addition, Xiao et al (7) used RNA-Seq data (GSE116250) and gene annotation of the Ensembl database to find the key module involved in DCM, and identified five co-expression modules that may have important functions in DCM occurrence. Thus, these previous studies provide possible research directions for elucidating the pathogenesis of DCM.

However, due to limited sample quantities, insufficient analysis methods and the lack of experimental verification, a number of previous DEG results for DCM may not be accurate (8). Therefore, to define the related genes in DCM, the present study performed an integrated bioinformatics analysis on four DCM microarrays from GEO, and a protein-protein interaction (PPI) network was established to investigate the interactions among DEGs. Then, the present study examined these hub genes in vivo using reverse transcription-quantitative PCR (RT-qPCR) in a mouse DCM model, which was established by intraperitoneal injection of doxorubicin (DOX).

\section{Materials and methods}

Raw transcriptional data acquisition and preprocessing. 'Dilated cardiomyopathy' and 'human' were used as key 
words to search the GEO database (http://www.ncbi.nlm. nih.gov/geo/), and raw data (CEL files) from five datasets (GSE17800, GSE21610, GSE42955, GSE79962 and GSE3585), consisting of myocardium transcriptional expression data from 89 patients with DCM and 37 healthy controls were downloaded (details in Table I) for further analysis. Raw data were first normalized using the RMA function in the R package affy (v1.64.0) (9). Probes in GSE17800, GSE21610 and GSE79962 were then screened by the MAS5CALLS algorithm packaged in affy, and probes detected as 'absent' across all samples were filtered out. The R packages hgu133plus2.db (v3.2.3) (10), hugene10sttranscriptcluster.db (v8.7.0) (11) and hgu133a.db (v3.2.3) (12) were used to annotate probes with gene symbols according to the microarray platform.

Meta-analysis of DEGs. The expression matrixes of GSE17800, GSE21610, GSE42955 and GSE79962 were merged by common gene symbols. MetaMA (v 3.1.2) (13) is an $\mathrm{R}$ package for microarray DEG meta-analyses that uses moderated effect size and P-value combinations, while RankProd (v3.12.0) (14) uses a non-parametric rank product method. The merged expression matrix was processed using metaMA and RankProd according to the reference manuals, and overlapping genes from the two methods were deemed as DEGs.

Functional annotation of the DEGs. Lists of upregulated DEGs and downregulated DEGs were uploaded to the Database for Annotation, Visualization and Integrated Discovery (DAVID; v6.8) $(15,16)$ website for Gene Ontology (GO, v2019-07) (17) and Kyoto Encyclopedia of Genes and Genomes (KEGG, v91.0) (18) pathway enrichment analysis. The results were downloaded from the website and then visualized using the R package ggplot2 (v3.2.0) (19).

PPI network construction. The DEG list was uploaded to the Search Tool for the Retrieval of Interacting Genes/Proteins (STRING; https://string-db.org/cgi/input.pl; v11.0) (20) database, and then a PPI network was established with the minimum required interaction score set as the highest confidence $(>0.9)$. Cytoscape (https://cytoscape.org, v3.7.1) was used to visualize the PPI network, and the MCODE (v1.6) (21) plug-in was used to screen important modules of the network.

Identification of hub genes. CytoHubba (v0.1) (22), a plug-in of Cytoscape software, was used to identify hub genes of the PPI network via four different algorithms. The algorithms used for analysis included degree, Maximum Neighborhood Component (MNC), Density of Maximum Neighborhood Component (DMNC) and Maximal Clique Centrality (MCC). A Venn diagram was constructed and consisted of genes ranked in the top 20 of each method, and genes overlapping in the four groups were deemed hub genes.

DCM animal model establishment. All animal studies were approved by the Animal Care and Ethics Committee of Nanjing Medical University. The animal experiments were performed according to the Guide for the Care and Use of Laboratory Animals (23). The DCM mouse model was established by intraperitoneal injection of DOX as previously described $(24,25)$. A total of 12 male C57BL/6 mice (age, 8 weeks; weight, 22-24 g) were purchased from Shanghai SLAC Laboratory Animal Co., Ltd. All mice were housed with sterile rodent chow and water available ad libitum in the animal holding room maintained at $26^{\circ} \mathrm{C}$ and $50 \%$ relative humidity, under a 12 -h light/dark cycle. Mice were randomly divided into two groups: Control $(n=6)$ and DOX $(n=6)$ groups. Mice in the DOX group were intraperitoneally injected with DOX hydrochloride (cat. no. HY-15142; MedChemExpress; $4 \mathrm{mg} / \mathrm{kg}$ ) once a week for 5 consecutive weeks. The control group received an equal volume $(0.1 \mathrm{ml})$ of normal saline $(0.9 \%)$. Mice that presented with progressive cardiac functional decline and ventricular dilation were successfully established as the DCM mouse model.

Transthoracic echocardiography. Then, 7 days after last injection, mice were sedated with $5 \%$ isoflurane- $\mathrm{O}_{2}$ (cat. no. 792632; Sigma-Aldrich; Merck KGaA) balanced mixture (maintained at 1.5\%). Transthoracic echocardiography (Vevo 1100; VisualSonics, Inc.) was performed in M-mode using a 30-MHz transducer immediately after anesthetization. The left ventricular (LV) echocardiogram was assessed in both parasternal long-axis and short-axis views. End-systolic and end-diastolic dimensions were defined as the phases corresponding to the electrocardiogram $\mathrm{T}$ wave and to the $\mathrm{R}$ wave, respectively (26). M-mode LV internal diameter end systole/diastole (LVIDs/d), LV posterior wall end diastole (LVPWd) and interventricular septal end diastole (IVSd) were averaged from 3-5 beats. Ejection fraction and fractional shortening were calculated as previously described (26).

$R N A$ extraction and $R T-q P C R$. After echocardiography detection all mice were anaesthetized by breathing a $5 \%$ isoflurane- $\mathrm{O}_{2}$ balanced mixture (maintained at 1.5\%). Mice were confirmed to be deeply anesthetized after they were immobile for $1 \mathrm{~min}$. Then, mice were euthanatized by inhalation of $25 \% \mathrm{CO}_{2}$, until respiratory and cardiac arrest occurred. Hearts were isolated and ground into $1 \mathrm{~mm}^{3}$ pieces, and then dissolved in pure TRI Reagent ${ }^{\circledR}$ (cat. no. 93289; Sigma-Aldrich; Merck KGaA). RNA was extracted using the RNAprep Pure Tissue kit (cat. no. DP431; Tiangen Biotech Co., Ltd.) according to the manufacturer's instructions. RT was performed using the PrimeScript RT reagent kit with gDNA Eraser (cat. no. RR047A; Takara Bio, Inc.) according to the manufacturer's instructions. gDNA was removed at $42^{\circ} \mathrm{C}$ for $2 \mathrm{~min}$, then cDNA was synthesized at $37^{\circ} \mathrm{C}$ for $15 \mathrm{~min}$ and $85^{\circ} \mathrm{C}$ for $5 \mathrm{sec}$. qPCR was conducted using the CellAmp Direct TB Green ${ }^{\circledast}$ RT-qPCR kit (cat. no. 3735A; Takara Bio, Inc.) and an ABI ViiA 7 system (cat. no. 4453536; Thermo Fisher Scientific, Inc.). The following thermocycling conditions were used: Melting at $95^{\circ} \mathrm{C}$ for $30 \mathrm{sec}$, annealing at $95^{\circ} \mathrm{C}$ for $3 \mathrm{sec}$, and extension at $60^{\circ} \mathrm{C}$ for $30 \mathrm{sec}$ for 40 cycles. The primers (Table II) for $\beta$-actin, proenkephalin (PENK), chordin like 1 (CHRDL1), calumenin (CALU), insulin-like growth factor binding protein 3 (IGFBP3) and ceruloplasmin (CP) were synthesized by Generay Biotech Co., Ltd. All samples were detected in triplicate, and gene expression values were normalized to the values of $\beta$-actin using $2^{-\Delta \Delta C q}$ method (27).

Statistical analysis. SPSS software (v16.0, SPSS, Inc.) was used for statistical analysis, and GraphPad Prism software 
Table I. Details of the microarrays used.

\begin{tabular}{|c|c|c|c|c|}
\hline GEO series number & Control & DCM & Tissue & Platform \\
\hline GSE17800 & 8 & 40 & Myocardium & $\begin{array}{l}\text { GPL570,[HG-U133_Plus_2] Affymetrix Human Genome U133 } \\
\text { Plus 2.0 Array }\end{array}$ \\
\hline GSE21610 & 8 & 21 & Myocardium & $\begin{array}{l}\text { GPL570,[HG-U133_Plus_2] Affymetrix Human Genome U133 } \\
\text { Plus 2.0 Array }\end{array}$ \\
\hline GSE42955 & 5 & 12 & Myocardium & $\begin{array}{l}\text { GPL6244,[HuGene-1_0-st] Affymetrix Human Gene 1.0 ST Array } \\
\text { [transcript (gene) version] }\end{array}$ \\
\hline GSE79962 & 11 & 9 & Myocardium & $\begin{array}{l}\text { GPL6244,[HuGene-1_0-st] Affymetrix Human Gene } 1.0 \text { ST Array } \\
\text { [transcript (gene) version] }\end{array}$ \\
\hline GSE3585 & 5 & 7 & Myocardium & GPL96,[HG-U133A] Affymetrix Human Genome U133A Array \\
\hline
\end{tabular}

GEO, Gene Expression Omnibus; DCM, dilated cardiomyopathy.

Table II. Primer sequences.

\begin{tabular}{lll}
\hline Gene & \multicolumn{1}{c}{ Forward $\left(5^{\prime} \rightarrow 3^{\prime}\right)$} & Reverse $\left(5^{\prime} \rightarrow 3^{\prime}\right)$ \\
\hline PENK & GGACTGCGCTAAATGCAGCTA & GAAGCCTCCGTACCGTTTCAT \\
CHRDL1 & AACCTCCAAGCCAAAACTTTGA & CCAGTGCTACTTTTCTGGTTGTC \\
CALU & AATGCTGATGGGTTCATTGATCT & GGTTCTTATCTCGAAACTCCACG \\
IGFBP3 & CACACCGAGTGACCGATTCC & GTGTCTGTGCTTTGAGACTCAT \\
CP & CTTAGCCTTGGCAAGAGATAAGC & GATGACAGGGCCTAAAAACCC \\
Actb & GGCTGTATTCCCCTCCATCG & CCAGTTGGTAACAATGCCATGT
\end{tabular}

PENK, proenkephalin; CHRDL1, chordin like 1; CALU, calumenin; IGFBP3, insulin-like growth factor binding protein 3; CP, ceruloplasmin; Actb, $\beta$-actin.

(v5, GraphPad Software, Inc.) was used for mapping. Data are presented as the mean \pm SD. For two-group comparisons, an independent sample t-test was used. $\mathrm{P}<0.05$ was considered to indicate a statistically significant difference.

\section{Results}

Identification of DEGs between patients with DCM and healthy controls. The raw data of GSE17800, GSE21610, GSE42955 and GSE79962, consisting of myocardium transcriptional expression data from 82 patients with DCM and 32 healthy controls, were downloaded from the GEO database. After preprocessing (Fig. 1A), the merged expression matrix of these four datasets was used for downstream analysis. The merged matrix was inputted into metaMA and RankProd, which are two $\mathrm{R}$ packages designed for meta-analysis of DEGs. A total of 1,943 genes (metaMA DEGs) were found to be differentially expressed by the metaMA package, while 1,390 genes (RankProd DEGs) were found to be differentially expressed by the RankProd package. A total of 898 overlapping genes between the metaMA DEGs and RankProd DEGs were considered DEGs (Fig. 1B), among which 424 genes were upregulated and 474 genes were downregulated (Fig. 1C). The top 30 upregulated genes and downregulated genes are listed in Tables III and IV, respectively. The most significantly upregulated genes were natriuretic peptide A (NPPA) and NPPB, while the most significantly downregulated gene was myosin heavy chain 6 (MYH6). In addition, the upregulation of NPPA and NPPB, and downregulation of MYH6 are well-established markers of heart dysfunction (28).

Validation of DEGs. To assess the robustness of the DEGs, GSE79962 was randomly selected for clustering heat map analysis with all 898 DEGs involved (Fig. 2A). For further validation, a fifth microarray dataset (GSE3585) out of the training data was downloaded. After normalization, filtering and annotation, the expression matrix of GSE3585 (healthy controls=5; patients with $\mathrm{DCM}=7$ ) was extracted. In total, 659 genes of the 898 DEGs were found in the GSE3585 expression matrix, and these 659 genes were used to plot a clustering heat map (Fig. 2B). These two clustering heat maps demonstrated that the DEGs were differentially expressed in the myocardium from patients with DCM compared with the healthy controls.

GO annotation of DEGs. To identify an overview of the biological functions of the DEGs, upregulated and downregulated DEGs were uploaded to the DAVID database for GO enrichment analysis. GO analysis results indicated that the upregulated genes were mainly involved in the following biological processes: Cell adhesion, negative regulation of cell proliferation, extracellular matrix (ECM) organization and 

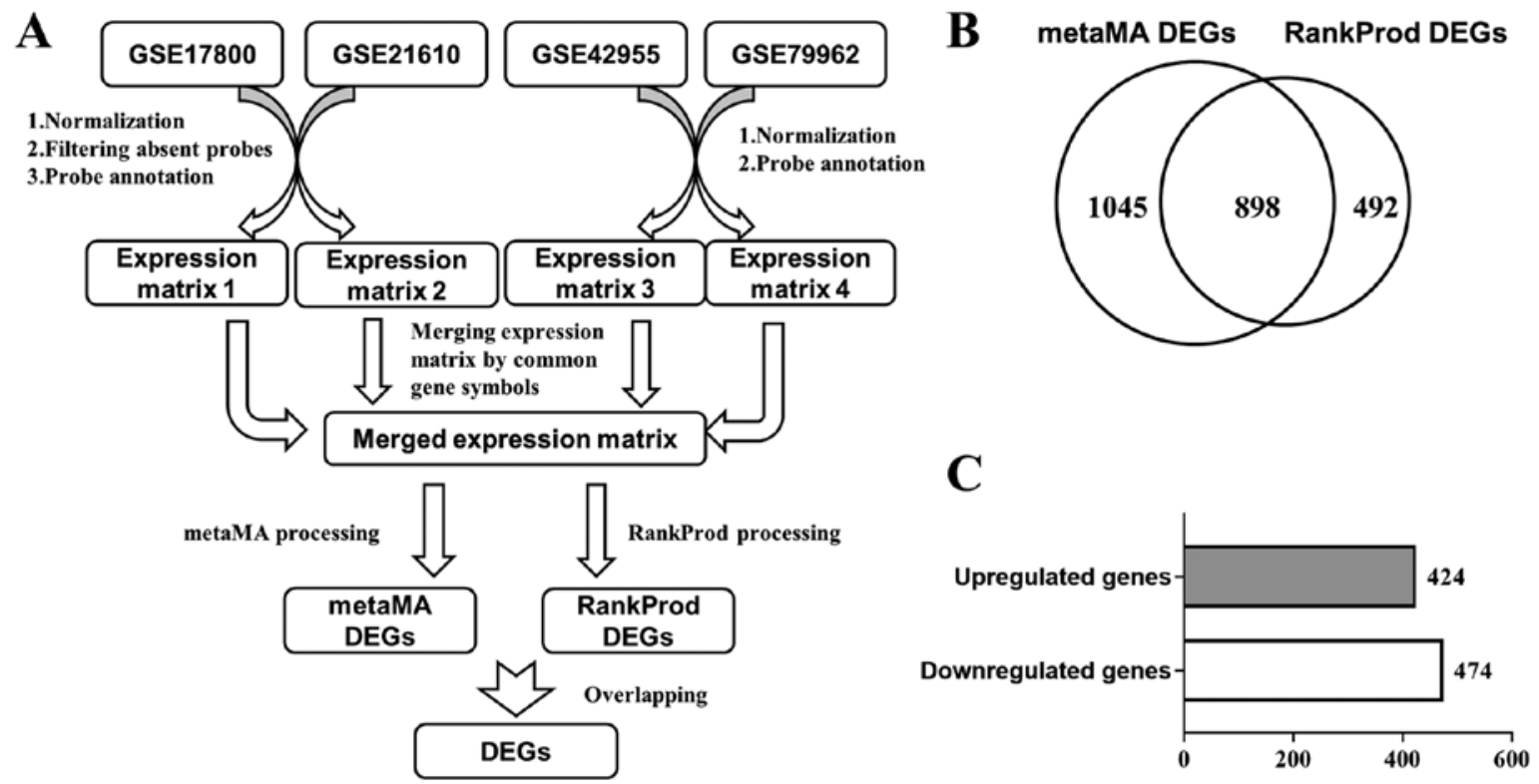

Figure 1. Identification of DEGs between patients with DCM and healthy controls. (A) Workflow identifying DEGs between patients with DCM and healthy controls. (B) Venn diagram of the DEGs outputted by metaMA and RankProd. A total of 1,943 DEGs were outputted by metaMA, while 1,390 DEGs were outputted by RankProd, among which 898 DEGs were overlapping. (C) Components of the DEGs. Among the 898 DEGs, 424 DEGs were upregulated and 474 DEGs were downregulated. DEGs, differentially expressed genes; DCM, dilated cardiomyopathy.

positive regulation of apoptotic process (Fig. 3A). In addition, downregulated genes were primarily enriched in the negative regulation of the apoptotic process, potassium ion transport, mitochondrial translational termination and mitochondrial translational elongation (Fig. 3B). Furthermore, all these biological processes are closely related to the occurrence and development of DCM. In addition, it was revealed that both the upregulated and downregulated genes were involved in the process of apoptosis regulation, which indicates the importance of apoptosis in DCM.

KEGG pathway enrichment of the DEGs. The present study investigated the KEGG pathways involved in the DEGs. It was demonstrated that upregulated DEGs were mainly enriched in the ECM-receptor interaction, the p53 signaling pathway, focal adhesion and the transforming growth factor- $\beta$ signaling pathway (Fig. 3C). In addition, downregulated DEGs were primarily enriched in cardiac muscle contraction, the hypoxia-inducible factor-1 signaling pathway, adrenergic signaling in cardiomyocytes and the cGMP-protein kinase G signaling pathway (Fig. 3D). Therefore, the present results indicated that these pathways may play vital roles in the progression of DCM.

Construction of the PPI network, and identification of important modules and hub genes. To assess the interactions among the proteins encoded by the DEGs, DEGs were uploaded to the STRING database to establish the PPI network (Fig. 4). It was demonstrated that there were 300 nodes and 852 edges in the network. Furthermore, among the 300 nodes, 151 nodes were upregulated, while 149 were downregulated. The MCODE plug-in was used to identify key modules of the PPI network, and the top three modules are presented in Fig. 5A-C. GO analysis results indicated that DEGs in module 2 were mainly enriched in protein ubiquitination-related biological processes, while the DEGs in module 3 were collagen- and ECM-related (Fig. 5D). Using four algorithms, the cytoHubba plug-in was used to detect hub genes of the PPI network. In addition, the top 20 genes were identified by degree, MCC, MNC and DMNC, which were then used for Venn diagram analysis (Fig. 5E). It was revealed that PENK, CHRDL1, CALU, apolipoprotein L1 (APOL1), IGFBP3 and CP overlapped among the four groups, and thus were deemed the hub genes that may play important roles in DCM (Table V).

Validation of the hub genes in a mouse DCM model. Mice receiving repeated DOX injections were used as a DCM in vivo model. As previously reported $(29,30)$, DOX mice that presented with progressive cardiac functional decline and ventricular dilation were successfully established as the DCM mouse model. Mice injected with cumulative $20 \mathrm{mg} / \mathrm{kg}$ DOX exhibited impaired heart function (Fig. 6A-C), dilated LV (Fig. 6D and E) and thin ventricular walls (Fig. 6F and G), which imitated the pathological manifestation of human DCM (31). APOL1 does not have a homologous gene in mice (32), but the mRNA expression levels of PENK, CHRDL1, CALU, IGFBP3 and CP in mouse hearts were detected with RT-qPCR. The present results indicated that the mRNA expression levels of PENK, CHRDL1, IGFBP3 and CP were significantly increased, and the expression of CALU was significantly decreased, in the hearts of mice treated with DOX compared with the saline controls (Fig. 6H). In addition, the changes in the expression of these hub genes observed in vivo were consistent with the microarray data, thus indicating an potential connection between hub genes and DCM.

\section{Discussion}

The present study analyzed four DCM microarrays from GEO, which to the best of the authors' knowledge is the largest sample 
Table III. Top 30 upregulated differentially expressed genes.

\begin{tabular}{|c|c|c|}
\hline $\begin{array}{l}\text { Upregulated } \\
\text { gene }\end{array}$ & $\begin{array}{c}\text { Fold } \\
\text { change }\end{array}$ & $\begin{array}{l}\text { Percentage of } \\
\text { false prediction }\end{array}$ \\
\hline NPPA & 5.57 & $6.41 \times 10^{-49}$ \\
\hline NPPB & 4.60 & $5.28 \times 10^{-40}$ \\
\hline SFRP4 & 2.46 & $2.61 \times 10^{-25}$ \\
\hline SMOC2 & 2.28 & $4.41 \times 10^{-23}$ \\
\hline $\mathrm{HBB}$ & 2.53 & $1.40 \times 10^{-22}$ \\
\hline EIF1AY & 2.45 & $1.82 \times 10^{-22}$ \\
\hline PHLDA1 & 1.99 & $4.61 \times 10^{-22}$ \\
\hline LTBP2 & 2.06 & $7.48 \times 10^{-20}$ \\
\hline THBS4 & 2.05 & $2.12 \times 10^{-19}$ \\
\hline FRZB & 2.07 & $5.42 \times 10^{-19}$ \\
\hline POSTN & 1.91 & $5.71 \times 10^{-19}$ \\
\hline MXRA5 & 1.82 & $1.01 \times 10^{-18}$ \\
\hline $\mathrm{CCN} 2$ & 1.96 & $6.47 \times 10^{-18}$ \\
\hline OMD & 2.00 & $6.13 \times 10^{-18}$ \\
\hline TNNT1 & 1.91 & $1.12 \times 10^{-17}$ \\
\hline PRELP & 2.06 & $1.34 \times 10^{-17}$ \\
\hline ASPN & 1.81 & $5.31 \times 10^{-17}$ \\
\hline FMOD & 1.94 & $7.19 \times 10^{-17}$ \\
\hline CCDC80 & 1.79 & $1.42 \times 10^{-16}$ \\
\hline RGS4 & 1.84 & $1.04 \times 10^{-15}$ \\
\hline SERPINE2 & 1.70 & $2.58 \times 10^{-15}$ \\
\hline USP9Y & 1.68 & $2.78 \times 10^{-15}$ \\
\hline STAT4 & 1.90 & $2.77 \times 10^{-15}$ \\
\hline PLCE1 & 1.75 & $2.67 \times 10^{-15}$ \\
\hline UCHL1 & 1.80 & $3.19 \times 10^{-15}$ \\
\hline OGN & 1.74 & $1.08 \times 10^{-14}$ \\
\hline HSPA2 & 1.72 & $2.10 \times 10^{-14}$ \\
\hline NAP1L3 & 1.77 & $3.88 \times 10^{-14}$ \\
\hline MFAP4 & 1.68 & $4.80 \times 10^{-14}$ \\
\hline JAK2 & 1.70 & $1.27 \times 10^{-13}$ \\
\hline
\end{tabular}

size that has been investigated, compared with the former researches on gene expression of $\operatorname{DCM}(6,7,33,34)$, using integrated bioinformatics analysis. With the establishment of a PPI network, six hub genes associated with DCM were identified, including upregulated PENK, CHRDL1, IGFBP3 and $\mathrm{CP}$, as well as downregulated CALU and APOL1. For further assessment of these hub genes, RT-qPCR was performed in a DOX-induced DCM mouse model. In line with the results of bioinformatics analysis, similar gene changes were observed in DOX mouse hearts; except for APOL1, which lacks homologous genes in mice (32). Furthermore, compared with mice in the control group, the expression levels of PENK, CHRDL1, IGFBP3 and CP were significantly increased in DOX mice, while the expression of CALU was significantly decreased.

It is speculated that some of the identified hub genes may serve as biological markers for early DCM screening in the clinic. $\mathrm{CP}$, encoding the metalloprotein that binds the copper in plasma, is involved in the peroxidation of $\mathrm{Fe}(\mathrm{II})$ transferrin to $\mathrm{Fe}(\mathrm{III})$ transferrin (35). In addition, $\mathrm{CP}$ is
Table IV. Top 30 downregulated differentially expressed genes.

\begin{tabular}{|c|c|c|}
\hline $\begin{array}{l}\text { Downregulated } \\
\text { gene }\end{array}$ & $\begin{array}{c}\text { Fold } \\
\text { change }\end{array}$ & $\begin{array}{l}\text { Percentage of } \\
\text { false prediction }\end{array}$ \\
\hline MYH6 & 0.32 & $2.33 \times 10^{-38}$ \\
\hline ETNPPL & 0.39 & $6.74 \times 10^{-29}$ \\
\hline SERPINA3 & 0.38 & $1.88 \times 10^{-27}$ \\
\hline DHRS7C & 0.45 & $1.51 \times 10^{-25}$ \\
\hline AQP4 & 0.52 & $1.29 \times 10^{-20}$ \\
\hline CCL2 & 0.47 & $1.89 \times 10^{-17}$ \\
\hline LYVE1 & 0.58 & $2.19 \times 10^{-16}$ \\
\hline LSAMP & 0.53 & $2.69 \times 10^{-16}$ \\
\hline DLK1 & 0.53 & $6.81 \times 10^{-16}$ \\
\hline CORIN & 0.53 & $1.04 \times 10^{-15}$ \\
\hline HOPX & 0.55 & $1.92 \times 10^{-15}$ \\
\hline CA14 & 0.55 & $4.94 \times 10^{-15}$ \\
\hline RARRES1 & 0.54 & $8.04 \times 10^{-15}$ \\
\hline CD163 & 0.62 & $9.97 \times 10^{-15}$ \\
\hline KCNIP2 & 0.60 & $1.01 \times 10^{-14}$ \\
\hline C1orf105 & 0.57 & $1.20 \times 10^{-14}$ \\
\hline CD14 & 0.56 & $3.45 \times 10^{-14}$ \\
\hline F13A1 & 0.61 & $1.53 \times 10^{-13}$ \\
\hline F5 & 0.82 & $2.01 \times 10^{-13}$ \\
\hline TOGARAM2 & 0.60 & $2.15 \times 10^{-13}$ \\
\hline CPNE4 & 0.59 & $2.05 \times 10^{-12}$ \\
\hline AQP3 & 0.62 & $4.89 \times 10^{-12}$ \\
\hline VSIG4 & 0.67 & $1.31 \times 10^{-11}$ \\
\hline HEY2 & 0.63 & $5.73 \times 10^{-11}$ \\
\hline PLIN2 & 0.62 & $6.50 \times 10^{-11}$ \\
\hline SELE & 0.57 & $1.46 \times 10^{-10}$ \\
\hline ADAMTS9 & 0.63 & $1.71 \times 10^{-10}$ \\
\hline NAMPT & 0.65 & $1.90 \times 10^{-10}$ \\
\hline SLCO5A1 & 0.60 & $2.63 \times 10^{-10}$ \\
\hline HINT3 & 0.60 & $9.40 \times 10^{-10}$ \\
\hline
\end{tabular}

associated with cardiovascular disease by decreasing nitric oxide bioavailability in blood (36). In a previous clinical study, CP was revealed to be independently related to cardiac function in patients with heart failure (37). CALU produces a $\mathrm{Ca}^{2+}$-binding protein that is localized in the endoplasmic reticulum (ER) (38). Furthermore, CALU is mainly expressed in the heart, and facilitates protein folding and sorting in the ER (38). During the excitation-contraction coupling process, CALU regulates $\mathrm{Ca}^{2+}$ uptake (39) and plays an important role in maintaining normal heart function (38). Thus, aberrant expression levels of CP and CALU could provide a foundation for the progressive decline of cardiac function in DCM, and have potential value in the early screening of DCM (40).

In addition, the DEGs identified in the present study may be valuable for the prognosis of DCM. PENK, encoding a preproprotein of pentapeptide opioids, mainly shows biased expression in adrenal tissue (41). Previous studies have also revealed that PENK is an important biomarker for renal dysfunction (42-44). In addition, APOL1, encoding a secreted 
A

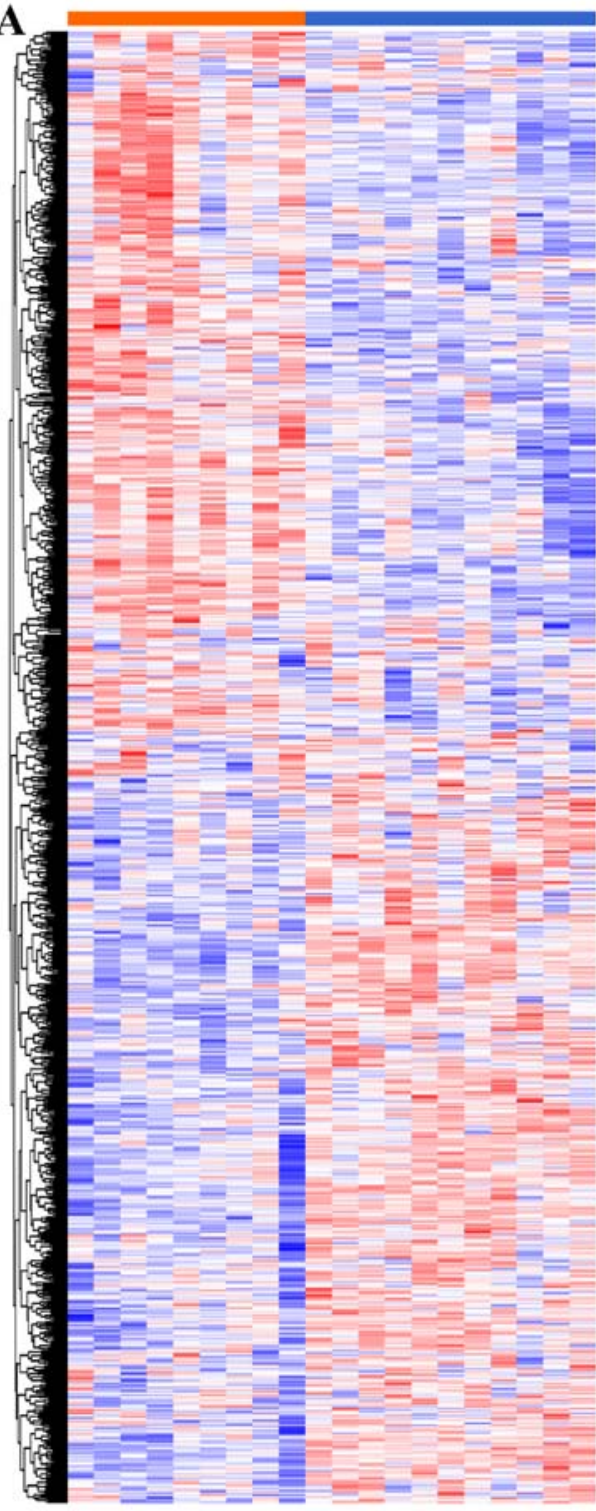

B

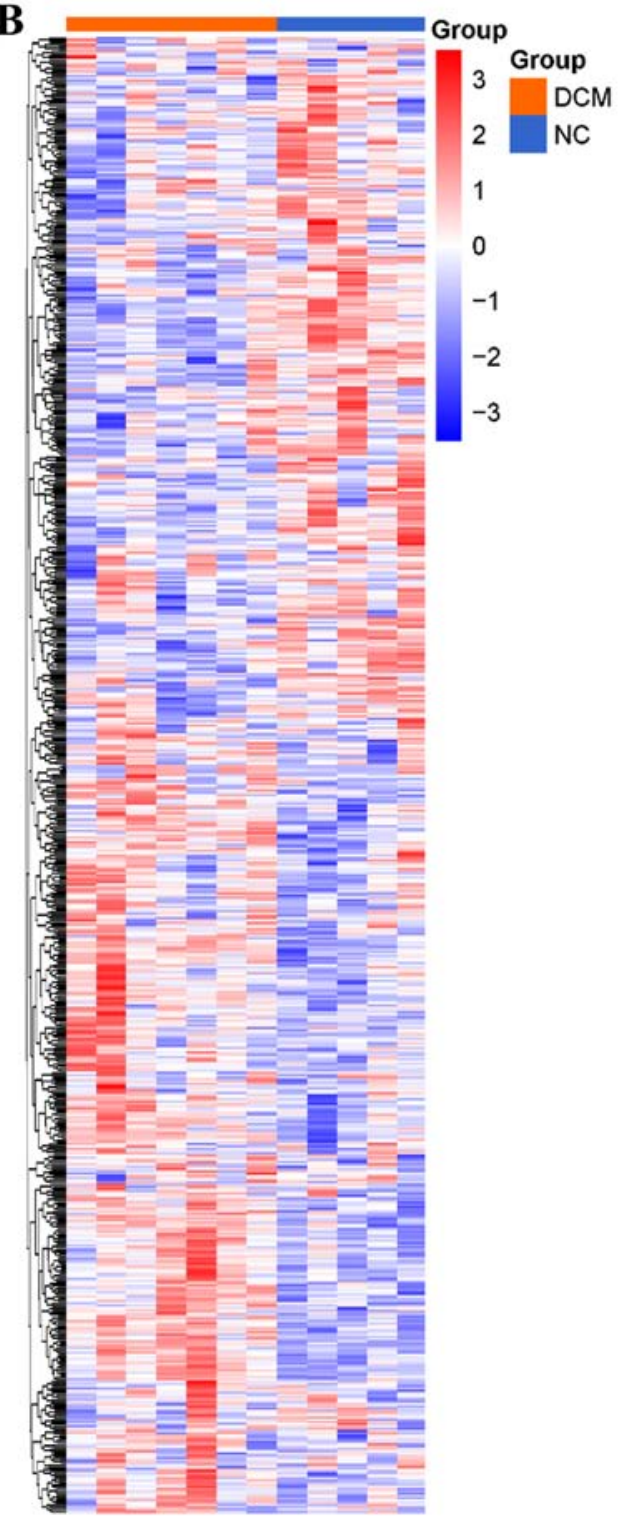

Figure 2. Validation of DEGs. (A) Clustering heat map of DEGs in GSE79962. (B) Clustering heat map of DEGs in GSE3585. The ordinate is the gene name, and the abscissa is the sample name. Red indicates high expression, while blue indicates low expression. Group DCM represents samples from patients with DCM, and NC represents samples from healthy controls. DEGs, differentially expressed genes; DCM, dilated cardiomyopathy; NC, healthy control.

high-density lipoprotein that binds to apolipoprotein A-I to promote lipid exchange, is reported to be associated with kidney disease when it is mutated (45). With regards to the interaction between the cardiovascular and renal systems, PENK and APOL1 are also of potential value for cardiac disease research (46). Kanagala et al (47) revealed a relationship between PENK and mortality rate in patients with heart failure. Furthermore, the aberrant expression of APOL1 has been revealed to play an important role in the development of hypertension (48). In line with these previous studies, the present results identified upregulation of PENK and downregulation of APOL1 in patients with DCM and DCM-induced mice, which indicated that these may provide prognostic information for the outcome in the progression of DCM.

Furthermore, the present study may provide a novel therapeutic target for the mechanism and treatment of DCM. CHRDL1 is an important gene that expresses the antagonist of bone morphogenetic protein 4 (BMP4) (49). In cardiac disease, BMP4 expression varies with cardiac function $(50,51)$. In addition, Wu et al (51) revealed that recombinant BMP4 plays a protective role in mouse cardiomyocytes. The present study found increased expression of CHRDL1 in DCM, which could potentially antagonize the protective effect of BMP4 and accelerate DCM progression. Thus, suppressing the expression of CHRDL1 may be a novel treatment target for attenuating DCM development. IGFBP3 is a member of the IGFBP family, and encodes a protein with an IGFBP domain and a thyroglobulin type-I domain (52). Previous studies have revealed that under hypoxic conditions, IGFBP3 can promote mitochondria-dependent cardiomyocyte apoptosis by inhibiting the IGF1R/PI3K/Akt survival pathway (53). In the present study, an increased expression of IGFBP3 was identified in DCM, indicating that aberrant IGFBP3 expression may be an important mechanism of DCM occurrence.

To investigate the identified hub genes, a DCM mouse model was established by intraperitoneal injection of DOX. 
A

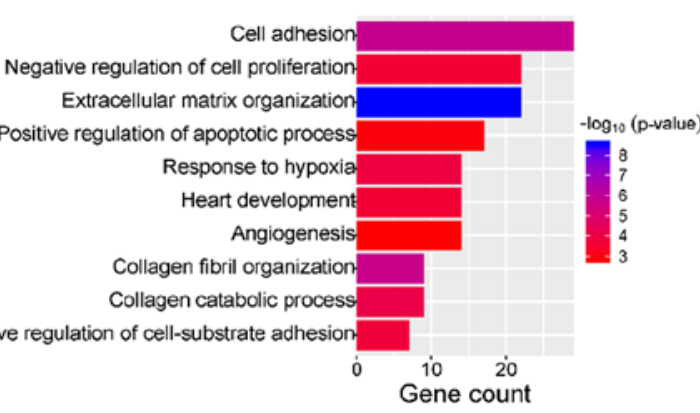

C

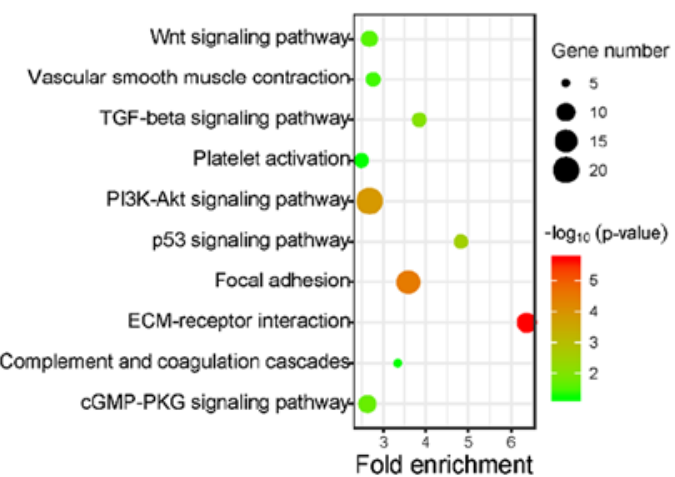

B

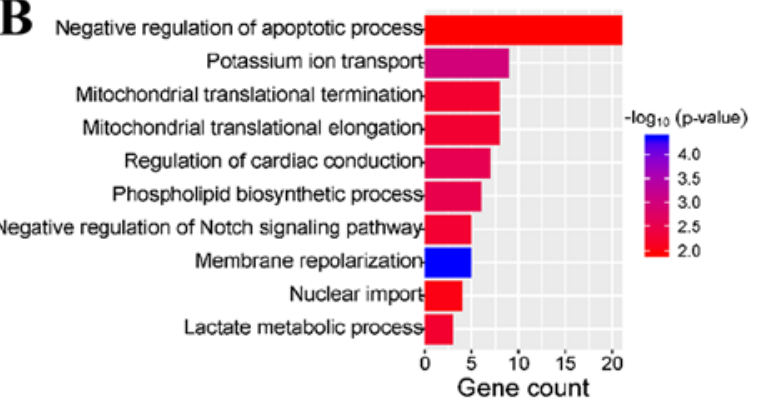

$\mathbf{D}$

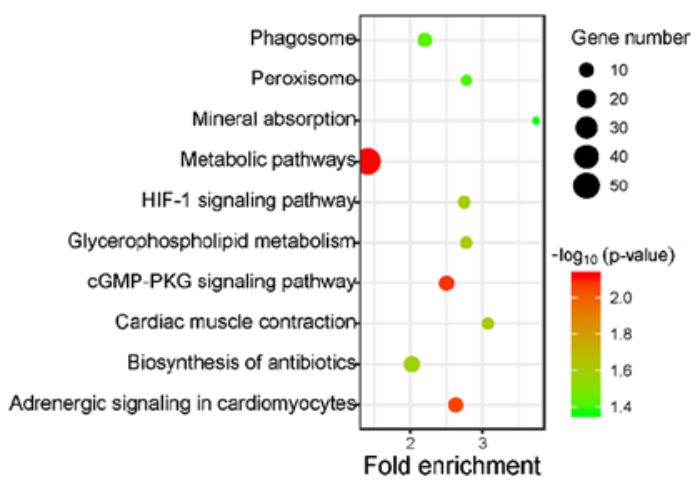

Figure 3. Functional annotation of DEGs. GO analysis of the (A) upregulated DEGs and (B) downregulated DEGs. The ordinate is the GO term name, and the abscissa is the count of DEGs enriched in the GO term. P-value of the enriched term is reflected by the color of the bar. KEGG pathway enrichment of the (C) upregulated DEGs and (D) downregulated DEGs. The ordinate is the name of the KEGG pathway, and the abscissa is the fold enrichment of the pathway. The count of DEGs is represented by bubble size and P-value by color. DEGs, differentially expressed genes; GO, Gene Ontology; KEGG, Kyoto Encyclopedia of Genes and Genomes; TGF- $\beta$, transforming growth factor $\beta$; ECM, extracellular matrix; PKG, protein kinase G.

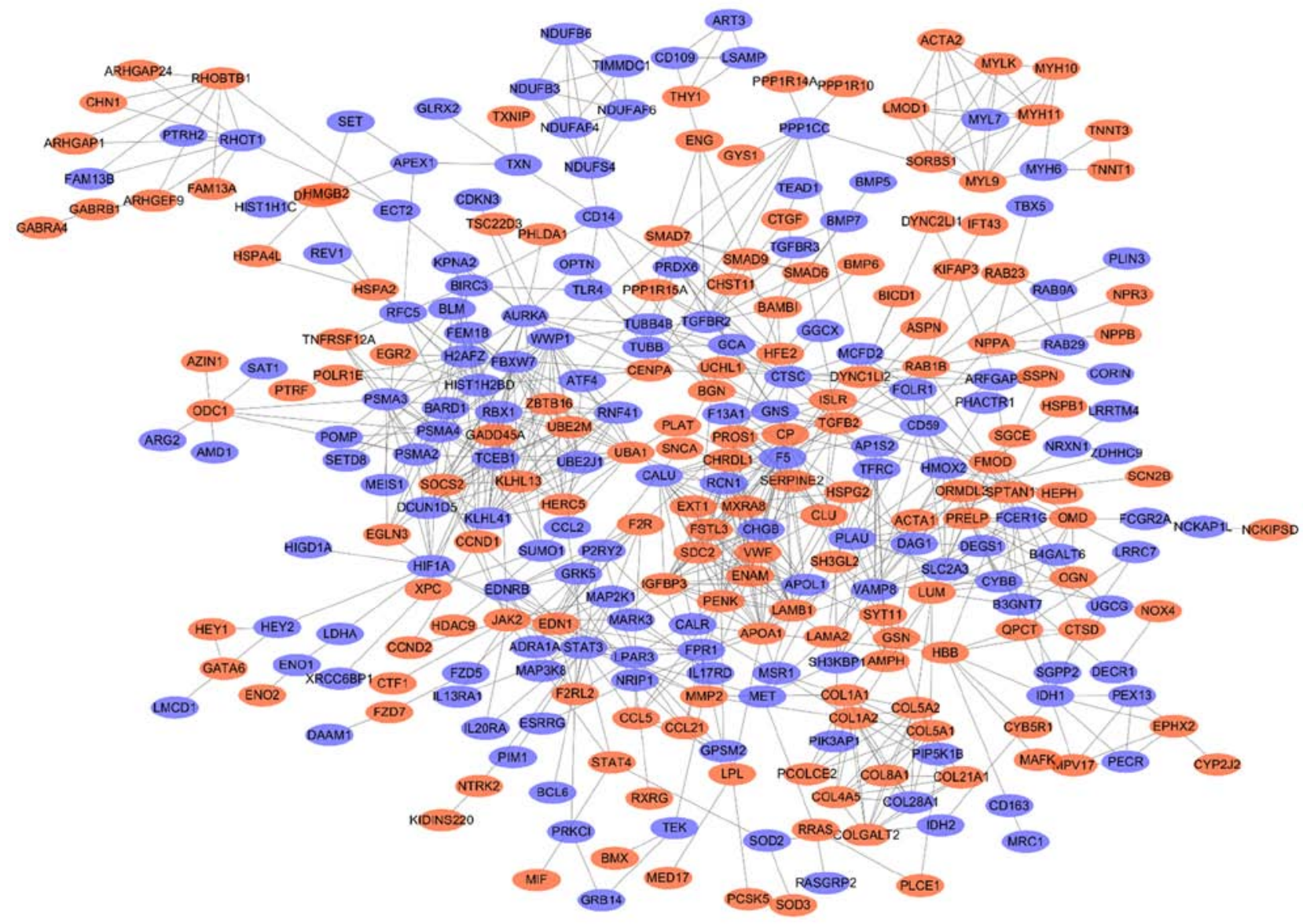

Figure 4. Construction of the PPI network. PPI network of DEGs. Red nodes are upregulated DEGs, while blue nodes are downregulated DEGs. Edges between nodes represent interactions of DEGs. DEGs, differentially expressed genes; PPI, protein-protein interaction. 


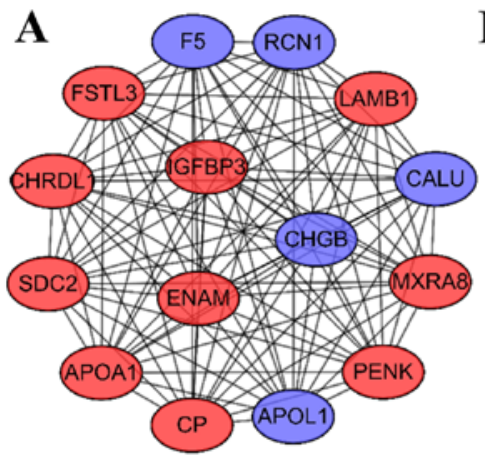

B

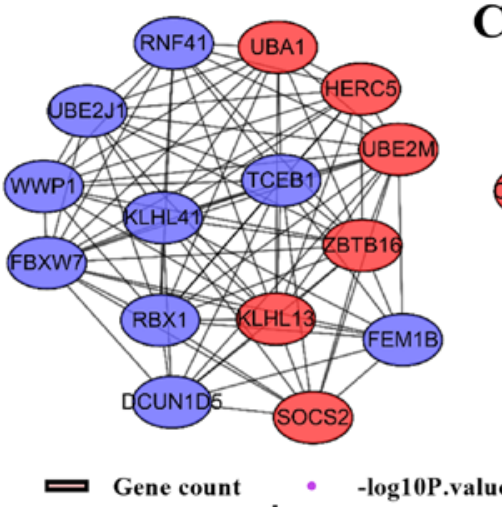

C

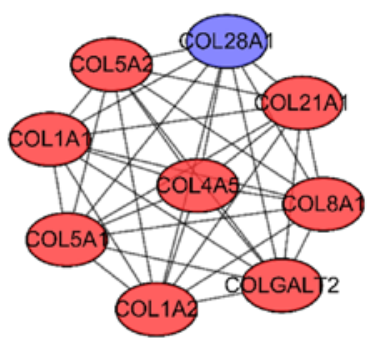

D

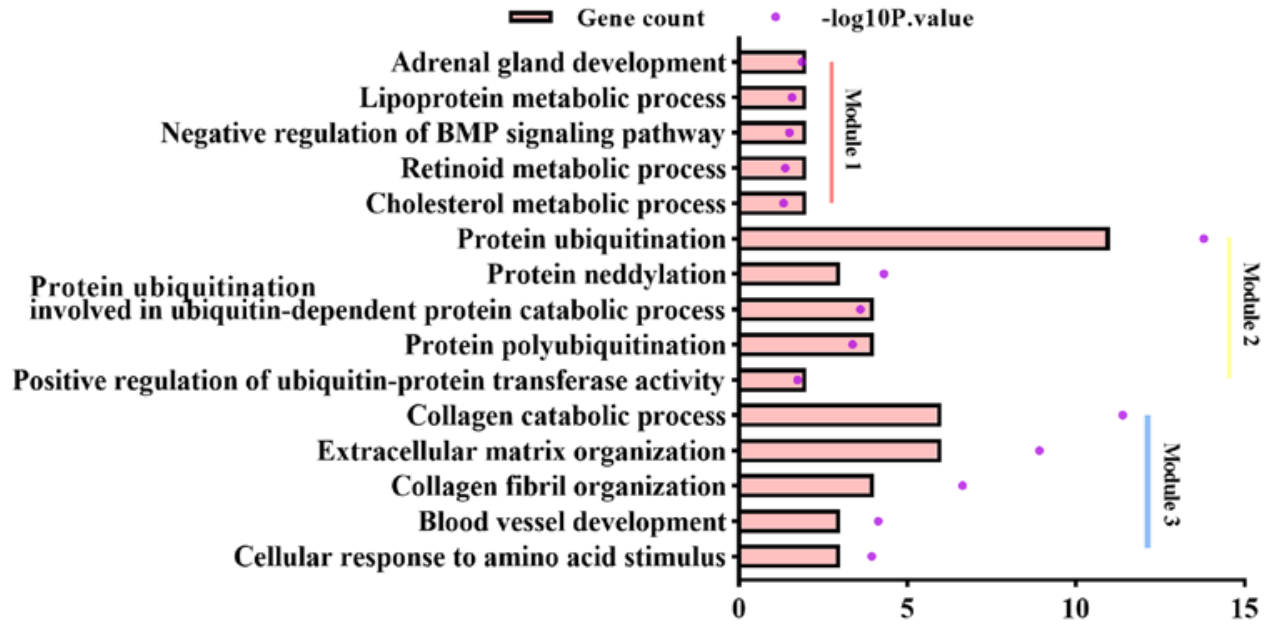

$\mathbf{E}$

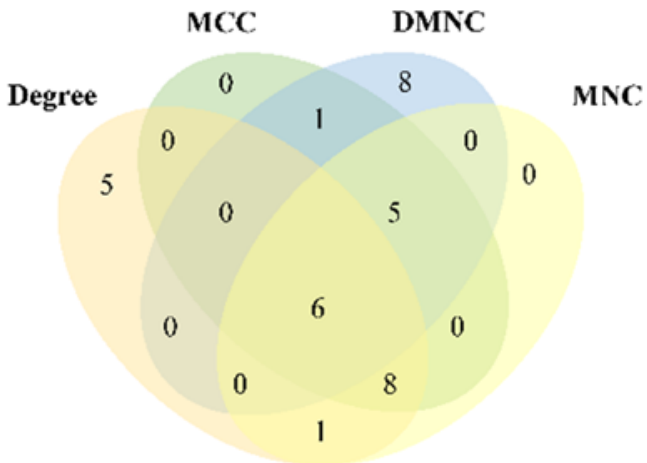

Figure 5. Identification of important modules and hub genes in the PPI network. (A) The first module, (B) the second module and (C) the third module of the PPI network detected by the MCODE plug-in and ranked from largest to smallest by score. Red nodes are upregulated DEGs, while blue nodes are downregulated DEGs. Edges between nodes represent interactions of DEGs. (D) GO analysis of the DEGs in module 1, module 2 and module 3 . The orange bar represents the count of DEGs enriched in the GO term, and the purple dot represents the -log10P-value. (E) Venn diagram of the top 20 genes calculated by four algorithms. DEGs, differentially expressed genes; PPI, protein-protein interaction; GO, Gene Ontology; BMP, bone morphogenetic protein; MNC, Maximum Neighborhood Component; DMNC, Density of Maximum Neighborhood Component; MCC, Maximal Clique Centrality.

DCM is the final common response of myocardium to diverse genetic and environmental insults (1). The etiological causes of DCM are various, and it is generally recognized that anthracycline cardiotoxicity is a common cause of DCM $(54,55)$. In addition, the clinical diagnosis of DCM depends on the clinical feature of progressively aggravated LV dilatation and systolic dysfunction (54). The DOX mouse model also exhibits progressive cardiac functional decline and ventricular dilation (29), which is consistent with the diagnosis of DCM. In addition, while the specific pathogenesis of DCM and the mechanism of its progressive progression remain unknown, previous studies revealed that the development of DCM may be associated with decreased mitochondrial function (56), abnormal oxidative stress $(57,58)$, as well as excessive myocardial apoptosis and necrosis $(59,60)$. In line with these possible causes, it has also been reported that the DOX model is accompanied by similar mitochondrial dysfunction $(29,61)$, oxidative stress injury (62) and apoptotic changes (63). Thus, the present study used the DOX mouse model to imitate the process of DCM for assessment of the hub genes. Consistent with the bioinformatics analysis results, similar genetic changes were observed in the DOX mouse model. It was determined that the expression levels of PENK, CHRDL1, IGFBP3 and CP were significantly increased in the hearts of 
Table V. Topological properties of hub genes.

\begin{tabular}{lccccccc}
\hline Hub gene & Degree & MCC & DMNC & MNC & Fold change & Percentage of false prediction & Expression change \\
\hline PENK & 19 & $8.72 \times 10^{10}$ & 1.02476 & 14 & 1.71 & $8.04 \times 10^{-13}$ & Upregulated \\
CHRDL1 & 16 & $8.72 \times 10^{10}$ & 1.02476 & 14 & 1.31 & $8.04 \times 10^{-6}$ & Upregulated \\
CALU & 16 & $8.72 \times 10^{10}$ & 0.92137 & 15 & 0.84 & $1.85 \times 10^{-2}$ & Downregulated \\
APOL1 & 15 & $8.72 \times 10^{10}$ & 0.92137 & 15 & 0.77 & $9.89 \times 10^{-3}$ & Downregulated \\
IGFBP3 & 15 & $8.72 \times 10^{10}$ & 0.92137 & 15 & 1.37 & $2.98 \times 10^{-6}$ & Upregulated \\
CP & 15 & $8.72 \times 10^{10}$ & 1.02476 & 14 & 1.32 & Upregulated \\
\hline
\end{tabular}

PENK, proenkephalin; CHRDL1, chordin like 1; CALU, calumenin; APOL1, apolipoprotein L1; IGFBP3, insulin-like growth factor binding protein 3; CP, ceruloplasmin; MNC, Maximum Neighborhood Component; DMNC, Density of Maximum Neighborhood Component; MCC, Maximal Clique Centrality.

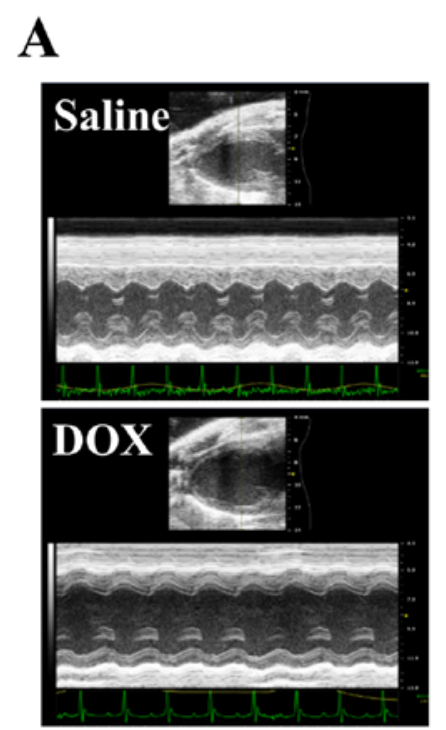

H
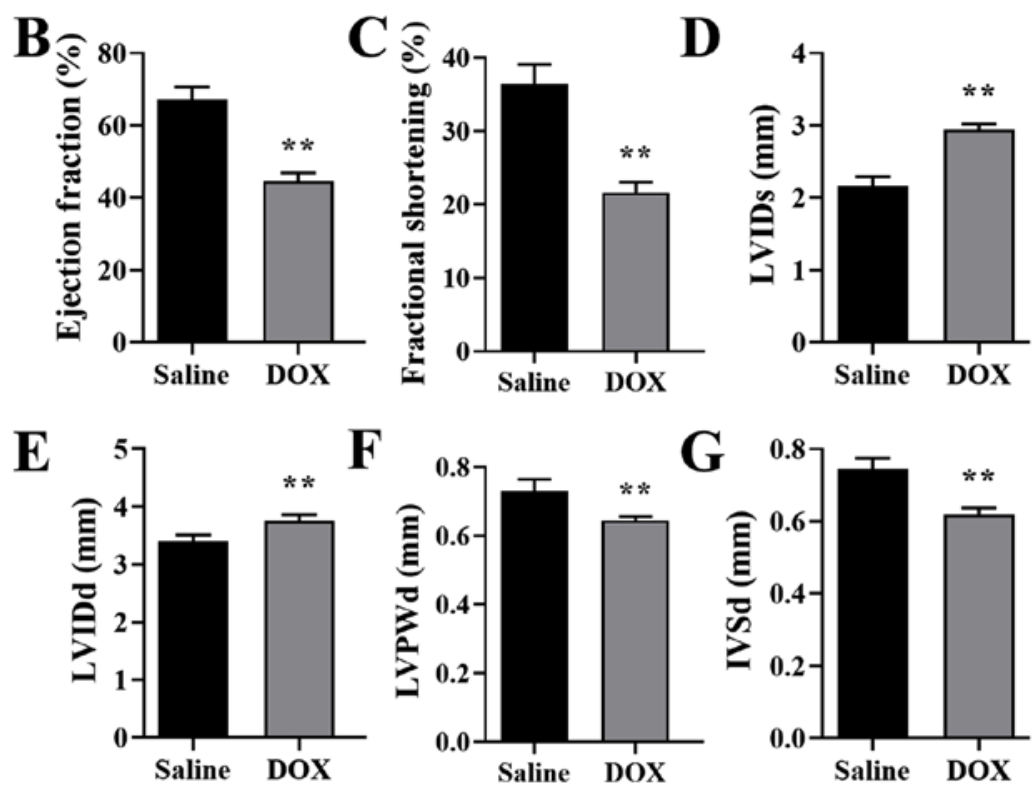

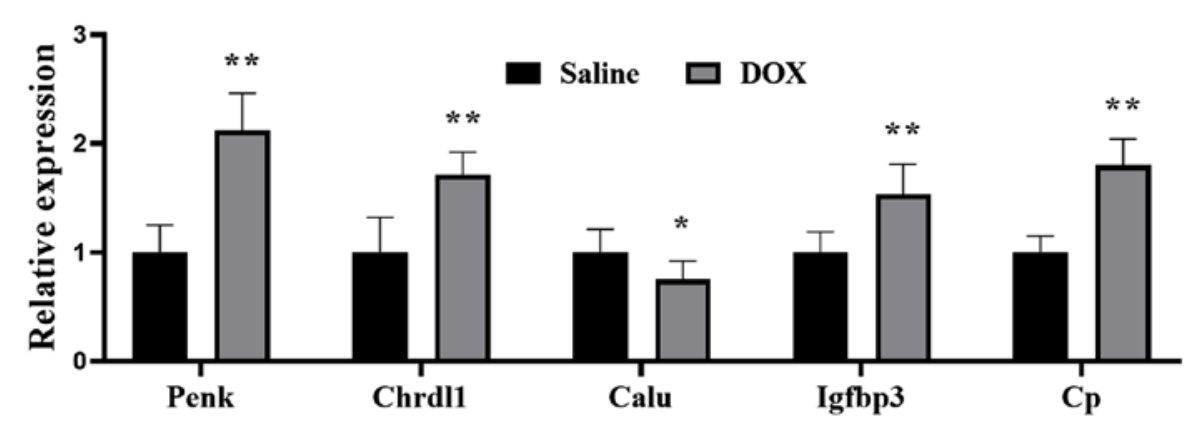

Figure 6. Validation of the hub genes in the mouse DCM model. (A) Representative echocardiography images of mice treated with saline and DOX. (B) Ejection fraction, (C) fractional shortening, (D) LVIDs, (E) LVIDd, (F) LVPWd and (G) IVSd of mice treated with saline or DOX. (H) Relative expression of PENK, CHRDL1, CALU, IGFBP3 and CP in the hearts of mice treated with DOX, normalized to the expression of $\beta$-actin. $\mathrm{n}=6$. ${ }^{*} \mathrm{P}<0.05$, ${ }^{* *} \mathrm{P}<0.01$ vs. saline. $\mathrm{DOX}$, doxorubicin; LVIDs/d, left ventricular internal diameter end systole/diastole; LVPWd, left ventricular posterior wall end diastole; IVSd, interventricular septal end diastole; PENK, proenkephalin; CHRDL1, chordin like 1; CALU, calumenin; IGFBP3, insulin-like growth factor binding protein 3; CP, ceruloplasmin.

DOX mice, while the expression of CALU was significantly decreased, which is consistent with the results of integrated microarray analysis.

However, while these hub genes in DCM have been identified, the specific underlying mechanisms of the genes involved in DCM development are not fully understood. Along with the involvement in autophagy, apoptosis and oxidation, which have been previously identified (64), these hub genes may also interact with each other, which further affects the survival and function of myocardial cells. Thus, further studies are still required to establish the causative correlations between these genes and the occurrence of DCM.

In conclusion, the present study identified six hub genes related to DCM, including PENK, CHRDL1, IGFBP3, CP, 
CALU and APOL1. In addition, these hub genes may provide a mechanism for DCM, and could serve as biomarkers for screening and diagnosis in the clinic.

\section{Acknowledgements}

Not applicable.

\section{Funding}

The present study was supported by the National Nature Science Foundation of China (grant no. 81770333).

\section{Availability of data and materials}

All data generated or analyzed during this study are included in this published article.

\section{Authors' contributions}

$\mathrm{HZ}, \mathrm{JH}$ and QS designed the present study, which was performed by JH, HZ and WJ. JH and WJ made substantial contributions to acquisition and analysis of data. $\mathrm{HZ}$ and WJ also made contributions to interpretation of data. $\mathrm{JH}$ wrote the initial draft of the manuscript. HZ revised it critically for important intellectual content. QS has given final approval of the version to be published. All authors have participated sufficiently in the work to take public responsibility for appropriate portions of the content and approved the manuscript, as well as agreed to be accountable for all aspects of the work. All authors read and approved the final manuscript.

\section{Ethics approval and consent to participate}

All animal studies were approved by the Animal Care and Ethics Committee of Nanjing Medical University. The animal experiments were performed according to the Guide for the Care and Use of Laboratory Animals (National Institutes of Health publication, 8th edition, 2011).

\section{Patient consent for publication}

Not applicable.

\section{Competing interests}

The authors declare that they have no competing interests.

\section{References}

1. Jefferies JL and Towbin JA: Dilated cardiomyopathy. Lancet 375 752-762, 2010.

2. Fatkin D, Huttner IG, Kovacic JC, Seidman JG and Seidman CE: Precision medicine in the management of dilated cardiomyopathy: JACC state-of-the-art review. J Am Coll Cardiol 74 2921-2938, 2019.

3. Richardson P, McKenna W, Bristow M, Maisch B, Mautner B, O'Connell J, Olsen E, Thiene G, Goodwin J, Gyarfas I, et al: Report of the 1995 world health organization/international society and federation of cardiology task force on the definition and classification of cardiomyopathies. Circulation 93: 841-842, 1996.
4. Hunt SA, Abraham WT, Chin MH, Feldman AM, Francis GS, Ganiats TG, Jessup M, Konstam MA, Mancini DM, Michl K, et al: 2009 Focused update incorporated into the ACC/AHA 2005 guidelines for the diagnosis and management of heart failure in adults a report of the American college of cardiology foundation/american heart association task force on practice guidelines developed in collaboration with the international society for heart and lung transplantation. J Am Coll Cardiol 53: e1-e90, 2009.

5. Rosenbaum AN, Agre KE and Pereira NL: Genetics of dilated cardiomyopathy: Practical implications for heart failure management. Nat Rev Cardiol 17: 286-297, 2020.

6. Huang H,Luo B, Wang B, Wu Q, Liang Y and He Y: Identification of potential gene interactions in heart failure caused by idiopathic dilated cardiomyopathy. Med Sci Monit 24: 7697-7709, 2018.

7. Xiao J, Li F, Yang Q, Zeng XF and Ke ZP: Co-expression analysis provides important module and pathways of human dilated cardiomyopathy. J Cell Physiol 235: 494-503, 2020.

8. Zhuang Y, Gong YJ, Zhong BF, Zhou Y and Gong L: Bioinformatics method identifies potential biomarkers of dilated cardiomyopathy in a human induced pluripotent stem cell-derived cardiomyocyte model. Exp Ther Med 14: 2771-2778, 2017.

9. Gautier L, Cope L, Bolstad BM and Irizarry RA: Affy-analysis of Affymetrix GeneChip data at the probe level. Bioinformatics 20: 307-315, 2004.

10. Carlson M: hgu133plus2.db: Affymetrix Human Genome U133 Plus 2.0 Array annotation data (chip hgu133plus2), R package version 3.2.3,2016.

11. MacDonald JW: hugene10sttranscriptcluster.db: Affymetrix hugene10 annotation data (chip hugene10sttranscriptcluster). $\mathrm{R}$ package version 8.7.0, 2017.

12. Carlson M: hgu133a.db: Affymetrix Human Genome U133 Set annotation data (chip hgu133a). R package version 3.2.3, 2016.

13. Marot G, Foulley JL, Mayer CD and Jaffrézic F: Moderated effect size and P-value combinations for microarray meta-analyses. Bioinformatics 25: 2692-2699, 2009.

14. Del Carratore F, Jankevics A, Eisinga R, Heskes T, Hong F and Breitling R: RankProd 2.0: A refactored bioconductor package for detecting differentially expressed features in molecular profiling datasets. Bioinformatics 33: 2774-2775, 2017.

15. Huang da W, Sherman BT and Lempicki RA: Systematic and integrative analysis of large gene lists using DAVID bioinformatics resources. Nat Protoc 4: 44-57, 2009.

16. Huang da W, Sherman BT and Lempicki RA: Bioinformatics enrichment tools: Paths toward the comprehensive functional analysis of large gene lists. Nucleic Acids Res 37: 1-13, 2009.

17. The Gene Ontology Consortium: The gene ontology resource: 20 years and still GOing strong. Nucleic Acids Res 47 (D1): D330-D338, 2019.

18. Kanehisa M, Furumichi M, Tanabe M, Sato Y and Morishima K: KEGG: New perspectives on genomes, pathways, diseases and drugs. Nucleic Acids Res 45(D1): D353-D361, 2017.

19. Wickham H: ggplot2: Elegant graphics for data analysis. Springer, 2016.

20. Szklarczyk D, Morris JH, Cook H, Kuhn M, Wyder S, Simonovic M, Santos A, Doncheva NT, Roth A, Bork P, et al: The STRING database in 2017: Quality-controlled protein-protein association networks, made broadly accessible. Nucleic Acids Res 45 (D1): D362-D368, 2017.

21. Bader GD and Hogue CW: An automated method for finding molecular complexes in large protein interaction networks. BMC Bioinformatics 4: 2, 2003.

22. Chin CH, Chen SH, Wu HH, Ho CW, Ko MT and Lin CY: cytoHubba: Identifying hub objects and sub-networks from complex interactome. BMC Syst Biol 8 (Suppl 4): S11, 2014.

23. National Research Council (US) Committee for the Update of the Guide for the Care and Use of Laboratory Animals: Guide for the Care and Use of Laboratory Animals, 8th edition, National Academies Press (US) National Academy of Sciences, Washington (DC), 2011.

24. Sun A, Cheng Y, Zhang Y, Zhang Q, Wang S, Tian S, Zou Y, $\mathrm{Hu}$ K, Ren J and Ge J: Aldehyde dehydrogenase 2 ameliorates doxorubicin-induced myocardial dysfunction through detoxification of 4-HNE and suppression of autophagy. J Mol Cell Cardiol 71: 92-104, 2014.

25. Xia Y, Chen Z, Chen A, Fu M, Dong Z, Hu K, Yang X, Zou Y, Sun A, Qian J and Ge J: LCZ696 improves cardiac function via alleviating Drp1-mediated mitochondrial dysfunction in mice with doxorubicin-induced dilated cardiomyopathy. J Mol Cell Cardiol 108: 138-148, 2017. 
26. Stypmann J, Engelen MA, Troatz C, Rothenburger M, Eckardt L and Tiemann K: Echocardiographic assessment of global left ventricular function in mice. Lab Anim 43: 127-137, 2009.

27. Livak KJ and Schmittgen TD: Analysis of relative gene expression data using real-time quantitative PCR and the 2(-Delta Delta C(T)) method. Methods 25: 402-408, 2001.

28. Gonzalez-Valdes I, Hidalgo I, Bujarrabal A, Lara-Pezzi E, Padron-Barthe L, Garcia-Pavia P, Gómez-del Arco P, Redondo JM, Ruiz-Cabello JM, Jimenez-Borreguero LJ, et al Bmil limits dilated cardiomyopathy and heart failure by inhibiting cardiac senescence. Nat Commun 6: 6473, 2015.

29. Li S, Wang W, Niu T, Wang H, Li B, Shao L, Lai Y, Li H, Janicki JS, Wang XL, et al: Nrf2 deficiency exaggerates doxorubicin-induced cardiotoxicity and cardiac dysfunction. Oxid Med Cell Longev 2014: 748524, 2014

30. Gomes AC, Falcão-Pires I, Pires AL, Brás-Silva C and Leite-Moreira AF: Rodent models of heart failure: An updated review. Heart Fail Rev 18: 219-249, 2013.

31. Bozkurt B, Colvin M, Cook J, Cooper LT, Deswal A, Fonarow GC, Francis GS, Lenihan D, Lewis EF, McNamara DM, et al: Current diagnostic and treatment strategies for specific dilated cardiomyopathies: A scientific statement from the American heart association. Circulation 134: e579-e646, 2016.

32. Bruggeman LA, Wu Z, Luo L, Madhavan SM, Konieczkowski M, Drawz PE, Thomas DB, Barisoni L, Sedor JR and O'Toole JF: APOL1-G0 or APOL1-G2 transgenic models develop preeclampsia but not kidney disease. J Am Soc Nephrol 27 : 3600-3610, 2016

33. Zhao J, Lv T, Quan J, Zhao W, Song J, Li Z, Lei H, Huang W and Ran L: Identification of target genes in cardiomyopathy with fibrosis and cardiac remodeling. J Biomed Sci 25: 63, 2018.

34. Zhang H, Yu Z, He J, Hua B and Zhang G: Identification of the molecular mechanisms underlying dilated cardiomyopathy via bioinformatic analysis of gene expression profiles. Exp Ther Med 13: 273-279, 2017.

35. Vasilyev VB: Looking for a partner: Ceruloplasmin in proteinprotein interactions. Biometals 32: 195-210, 2019.

36. Cabassi A, Binno SM, Tedeschi S, Ruzicka V, Dancelli S, Rocco R, Vicini V, Coghi P, Regolisti G, Montanari A, et al: Low serum ferroxidase I activity is associated with mortality in heart failure and related to both peroxynitrite-induced cysteine oxidation and tyrosine nitration of ceruloplasmin. Circ Res 114: 1723-1732, 2014

37. Hammadah M, Fan Y, Wu Y, Hazen SL and Tang WH: Prognostic value of elevated serum ceruloplasmin levels in patients with heart failure. J Card Fail 20: 946-952, 2014.

38. Sahoo SK and Kim do H: Characterization of calumenin in mouse heart. BMB Rep 43: 158-163, 2010.

39. Sahoo SK and Kim DH: Calumenin interacts with SERCA2 in rat cardiac sarcoplasmic reticulum. Mol Cells 26: 265-269, 2008.

40. Mazzarotto F, Tayal U, Buchan RJ, Midwinter W, Wilk A, Whiffin N, Govind R, Mazaika E, de Marvao A, Dawes TJW, et al: Reevaluating the genetic contribution of monogenic dilated cardiomyopathy. Circulation 141: 387-398, 2020

41. Ng LL, Squire IB, Jones DJ, Cao TH, Chan DCS, Sandhu JK, Quinn PA, Davies JE, Struck J, Hartmann O, et al: Proenkephalin, renal dysfunction, and prognosis in patients with acute heart failure: A GREAT network study. J Am Coll Cardiol 69: 56-69, 2017.

42. Hollinger A, Wittebole X, François B, Pickkers P, Antonelli M, Gayat E, Chousterman BG, Lascarrou JB, Dugernier T, Di Somma S, et al: Proenkephalin A 119-159 (Penkid) is an early biomarker of septic acute kidney injury: The kidney in sepsis and septic shock (Kid-SSS) study. Kidney Int Rep 3: 1424-1433, 2018.

43. Emmens JE, Ter Maaten JM, Damman K, van Veldhuisen DJ, de Boer RA, Struck J, Bergmann A, Sama IE, Streng KW, Anker SD, et al: Proenkephalin, an opioid system surrogate, as a novel comprehensive renal marker in heart failure. Circ Heart Fail 12: e005544, 2019.

44. Marino R, Struck J, Hartmann O, Maisel AS, Rehfeldt M, Magrini L, Melander O, Bergmann A and Di Somma S: Diagnostic and short-term prognostic utility of plasma pro-enkephalin (pro-ENK) for acute kidney injury in patients admitted with sepsis in the emergency department. J Nephrol 28 . 717-724, 2015

45. Shah S, Shapiro R, Murphy B and Menon MC: APOL1 high-risk genotypes and renal transplantation. Clin Transplant 33: e13582, 2019.

46. Yogasundaram H, Chappell MC, Braam B and Oudit GY: Cardiorenal syndrome and heart failure-challenges and opportunities. Can J Cardiol 35: 1208-1219, 2019.
47. Kanagala P, Squire IB, Jones DJL, Cao TH, Chan DCS, McCann G, Sandhu JK, Quinn PA, McAdam J, Marsh AM, et al: Proenkephalin and prognosis in heart failure with preserved ejection fraction: A GREAT network study. Clin Res Cardiol 108: 940-949, 2019.

48. Robinson TW and Freedman BI: The Impact of APOL1 on chronic kidney disease and hypertension. Adv Chronic Kidney Dis 26: 131-136, 2019.

49. Webb TR, Matarin M, Gardner JC, Kelberman D, Hassan H, Ang W, Michaelides M, Ruddle JB, Pennell CE, Yazar S, et al: $\mathrm{X}$-linked megalocornea caused by mutations in CHRDL1 identifies an essential role for ventroptin in anterior segment development. Am J Hum Genet 90: 247-259, 2012.

50. Sun B, Huo R, Sheng Y, Li Y, Xie X, Chen C, Liu HB, Li N, Li CB, Guo WT, et al: Bone morphogenetic protein-4 mediates cardiac hypertrophy, apoptosis, and fibrosis in experimentally pathological cardiac hypertrophy. Hypertension 61: 352-360, 2013.

51. Wu X, Sagave J, Rutkovskiy A, Haugen F, Baysa A, Nygård S, Czibik G, Dahl CP, Gullestad L, Vaage J and Valen G: Expression of bone morphogenetic protein 4 and its receptors in the remodeling heart. Life Sci 97: 145-154, 2014.

52. Ranke MB: Insulin-like growth factor binding-protein-3 (IGFBP-3). Best Pract. Res Clin Endocrinol Metab 29: 701-711, 2015.

53. Feng CC, Lin CC, Lai YP, Chen TS, Marthandam Asokan S, Lin JY, Lin KH, Viswanadha VP, Kuo WW and Huang CY: Hypoxia suppresses myocardial survival pathway through HIF-1 $\alpha$-IGFBP-3-dependent signaling and enhances cardiomyocyte autophagic and apoptotic effects mainly via FoxO3a-induced BNIP3 expression. Growth Factors 34: 73-86, 2016.

54. Japp AG, Gulati A, Cook SA, Cowie MR and Prasad SK: The diagnosis and evaluation of dilated cardiomyopathy. J Am Coll Cardiol 67: 2996-3010, 2016.

55. Ky B, Putt M, Sawaya H, French B, Januzzi JL Jr, Sebag IA, Plana JC, Cohen V, Banchs J, Carver JR, et al: Early increases in multiple biomarkers predict subsequent cardiotoxicity in patients with breast cancer treated with doxorubicin, taxanes, and trastuzumab. J Am Coll Cardiol 63: 809-816, 2014.

56. Chen L and Knowlton AA: Mitochondrial dynamics in heart failure. Congest Heart Fail 17: 257-261, 2011.

57. Pang XF, Lin X, Du JJ and Zeng DY: LTBP2 knockdown by siRNA reverses myocardial oxidative stress injury, fibrosis and remodelling during dilated cardiomyopathy. Acta Physiol (Oxf) 228: e13377, 2020.

58. Lubrano V and Balzan S: Role of oxidative stress-related biomarkers in heart failure: Galectin 3, 1 -antitrypsin and LOX-1: New therapeutic perspective? Mol Cell Biochem 464: $143-152,2020$

59. Liu L, Sun K, Zhang X, Tang Y and Xu D: Advances in the role and mechanism of BAG3 in dilated cardiomyopathy. Heart Fail Rev, Dec 6, 2019 (Epub ahead of print).

60. Mazelin L, Panthu B, Nicot AS, Belotti E, Tintignac L, Teixeira G, Zhang Q, Risson V, Baas D, Delaune E, et al: mTOR inactivation in myocardium from infant mice rapidly leads to dilated cardiomyopathy due to translation defects and p53/JNK-mediated apoptosis. J Mol Cell Cardiol 97: 213-225, 2016.

61. Ni C, Ma P, Wang R, Lou X, Liu X, Qin Y, Xue R, Blasig I, Erben U and Qin Z: Doxorubicin-induced cardiotoxicity involves IFN $\gamma$-mediated metabolic reprogramming in cardiomyocytes. J Pathol 247: 320-332, 2019.

62. Goyal V, Bews H, Cheung D, Premecz S, Mandal S, Shaikh B, Best R, Bhindi R, Chaudhary R, Ravandi A, et al: The cardioprotective role of $\mathrm{N}$-Acetyl cysteine amide in the prevention of doxorubicin and trastuzumab-mediated cardiac dysfunction. Can J Cardiol 32: 1513-1519, 2016.

63. Liu D, Ma Z, Di S, Yang Y, Yang J, Xu L, Reiter RJ, Qiao S and Yuan J: AMPK/PGC1 $\alpha$ activation by melatonin attenuates acute doxorubicin cardiotoxicity via alleviating mitochondrial oxidative damage and apoptosis. Free Radic Biol Med 129: 59-72, 2018.

64. Schultheiss HP, Fairweather D, Caforio ALP, Escher F, Hershberger RE, Lipshultz SE, Liu PP, Matsumori A, Mazzanti A, McMurray J and Priori SG: Dilated cardiomyopathy. Nat Rev Dis Primers 5: 32, 2019.

This work is licensed under a Creative Commons Attribution-NonCommercial-NoDerivatives 4.0 International (CC BY-NC-ND 4.0) License. 